\title{
Shaping zoonosis risk: landscape ecology vs. landscape attractiveness for people, the case of tick-borne encephalitis in Sweden
}

\author{
Caroline B Zeimes $^{1 *}$, Gert E Olsson², Marika Hjertqvist ${ }^{3}$ and Sophie O Vanwambeke ${ }^{1}$
}

\begin{abstract}
Background: In this paper, the hazard and exposure concepts from risk assessment are applied in an innovative approach to understand zoonotic disease risk. Hazard is here related to the landscape ecology determining where the hosts, vectors and pathogens are and, exposure is defined as the attractiveness and accessibility to hazardous areas. Tick-borne encephalitis in Sweden was used as a case study.

Methods: Three boosted regression tree models are compared: a hazard model, an exposure model and a global model which combines the two approaches.

Results: The global model offers the best predictive power and the most accurate modelling. The highest probabilities were found in easy-to-reach places with high landscape diversity, holiday houses, waterbodies and, well-connected forests of oak, birch or pine, with open-area in their ecotones, a complex shape, numerous clear-cuts and, a variation in tree height.

Conclusion: While conditions for access and use of hazardous areas are quite specific to Scandinavia, this study offers promising perspectives to improve our understanding of the distribution of zoonotic and vector-borne diseases in diverse contexts.
\end{abstract}

Keywords: Tick-borne disease, Zoonoses, Vector-borne disease, Risk

\section{Background}

Most emerging diseases are of zoonotic origin [1]. As they involve pathogens, hosts and, potentially vectors, zoonoses are complex disease systems and a challenge for public health. In this paper, concepts of risk assessment are applied to a vector-borne zoonotic disease in an innovative approach to untangle sources of risk. Risk assessment includes the identification of hazard and the characterization of exposure [2]. The hazard is any potential source of damage (e.g. radioactive radiation), while the exposure is the chance that populations will be in contact with the hazard (e.g. work in a nuclear power plant). In the context of zoonotic vector-borne diseases, we define hazard as the number of infected hosts or vectors in the environment. This is determined

\footnotetext{
*Correspondence: caroline.zeimes@uclouvain.be

'Earth and Life Institute, Georges Lemaître Centre for Earth and Climate Research (TECLIM), Université Catholique de Louvain (UCL), GEOG, Place Louis Pasteur 3 bte L4.03.07, 1348 Louvain-la-Neuve, Belgium

Full list of author information is available at the end of the article
}

by ecological conditions allowing the hosts, vectors and pathogens to complete their life cycles and to overlap. Exposure concerns the degree to which humans get in contact with infected hosts/vectors. This largely relates to land use, including the ability and the attraction to access places where infected hosts/vectors are found. Many disease ecology studies focus on what is here defined as hazard. Exposure is more commonly addressed by the field of public health which often does not include landscape-related variables. However, the distribution of disease cases potentially results from the combination of both hazard and exposure, and therefore cannot be approached solely from the hazard angle. In this study, we attempt to distinguish between hazard and exposure by comparing the predictive power of three models that focus on different aspects of the landscape: a hazard model containing a set of variables found in the ecological literature, an exposure model containing a set of variables found in the 
touristic and public health literature and, a global model containing both sets of variables. Tick-borne encephalitis (TBE) in Sweden is used as a case study. TBE has already been well studied under the hazard angle (e.g. [3]) but less under the angle of exposure (which is emphasized in [4]).

TBE virus (TBEV) belongs to the family of Flaviviruses and the western subtype of TBEV is usually transmitted by Ixodes ricinus ticks [5]. Ticks pass through three active life stages (larvae, nymphs and adults) and need a blood meal to reach the following respective stage [6]. Transmission of TBEV among ticks occurs mainly during co-feeding, especially between uninfected larva and infected nymph feeding on rodents [7-9].

TBEV is of concern in Sweden, as the tick population has spread and the incidence of the disease has been increasing sharply over the past few years [10]. Two phenomena are currently observed in Sweden. On the one hand, the range of human cases of $\mathrm{TBE}$ is expanding westward within the known tick range, and on the other hand, the expansion of ticks northward along the coast. While some common factors may be at play, the mechanisms behind each phenomenon have not been fully clarified. This may result from climate changes, host populations dynamics and human behaviour changes $[11,12]$. However, even in the well-established TBEV endemic areas around Stockholm, the effect of these variables on spatial distribution of the disease is unclear.

\section{Methods}

\section{Materials}

The study focused on Stockholm and the five neighbouring counties (Gävleborgs län, Dalarnas län, Uppsala län, Västmanlands län and Södermanlands län) (Figure 1). TBEV is well established in that region of Sweden, where the disease has been recorded for the past century. Records of cases by nearest settlement of infection (SMI Swedish Institute for Communicable Disease Control (Smittskyddsinstitutet) were included for a ten-year study period (January 1998 to December 2007). Presence at any time during the study period was translated into a presence record, totaling 125 presence records. The other settlements extracted from the Lantmäteriet database (Swedish mapping, cadastral and land registration authority), constituted the 4297 absence records.

Potential explanatory variables either represented the surrounding environment, as calculated in a radius of two $\mathrm{km}$ around the point location, or were calculated at the exact record location.

Hypotheses were made based on literature, and candidate explanatory variables were allocated to hazard or exposure. However, some variables could not be allocated to

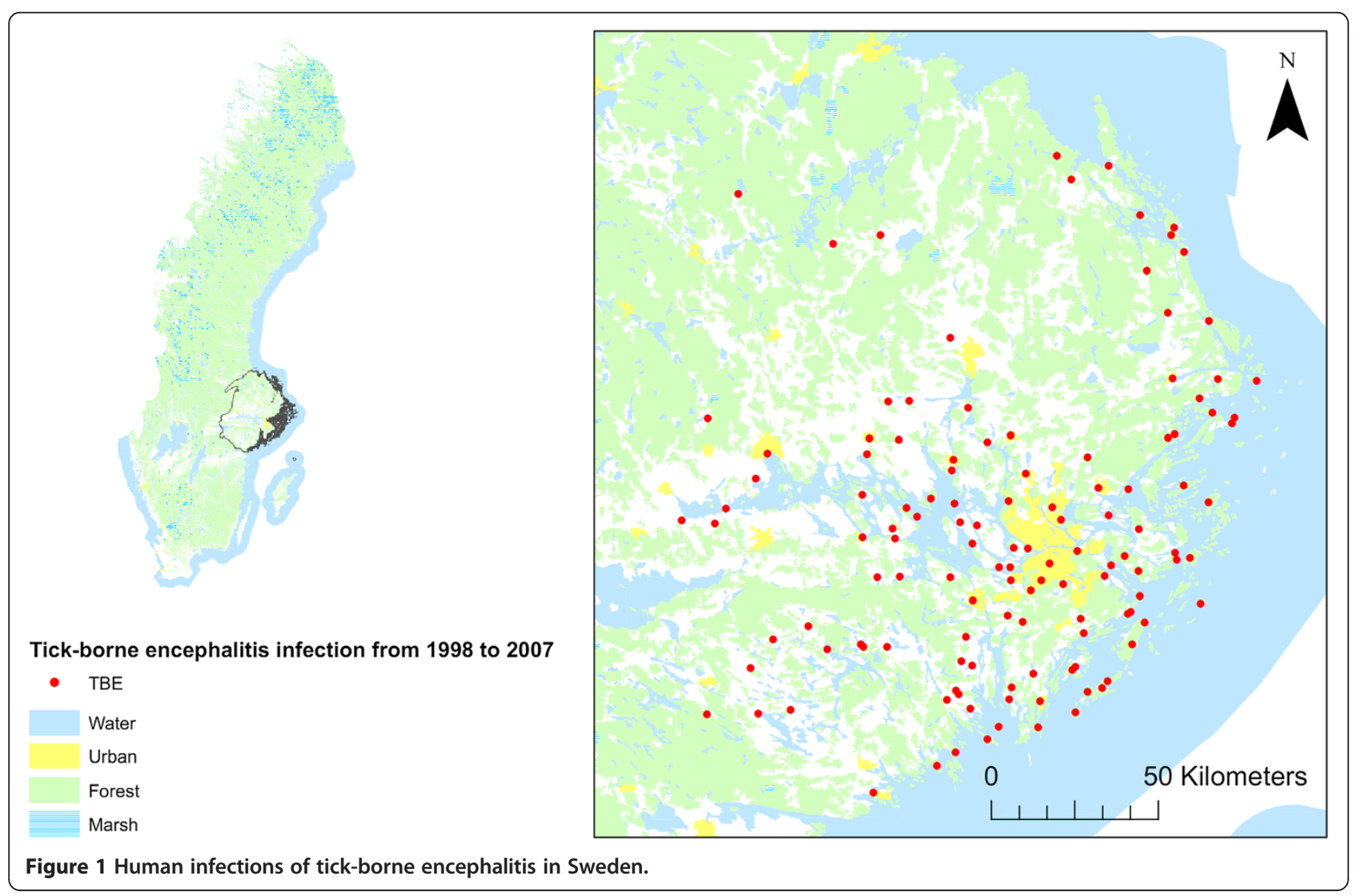


a single group in an unequivocal way and were included in both.

\section{Variables describing hazard}

Variables describing the hazard, places where infected hosts or vectors are found, were identified in the literature (Table 1). They are linked to the ecology of the hosts and vector. Some commonly used landscape metrics were also included.

\section{i. Animal species}

In Sweden, roe deer are a major blood meal host for reproducing adult female ticks $[11,13]$. However, other larger game species, e.g. red deer, fallow deer and wild boar, are available in large numbers and are also likely to be important hosts for ticks. Bag records of these game species were included ( $\mathrm{Dr}$ Jonas Kindberg, Wildlife Monitoring Unit, Swedish Association for Hunting and Wildlife Management, personal communication). Data, available by centre of hunting districts, were interpolated by Thiessen polygons, leading to a lower resolution compared to the other variables. The number of animals per hectare found at the point is used as a proxy for blood meal availability.

ii. Forest

Deciduous forests are a highly suitable habitat for ticks, as well as for some host mammals $[14,15]$. The total proportion of forest, broad-leaved forest, conifer and mixed forest inside the two km buffer (100 m resolution, CORINE Land Cover, EEA) were included. The average shape and proximity index of forest patches in the buffer were calculated. A patch with the most compact shape (i.e. the smallest patch to area ratio), in the case of raster data, a square, has a shape index of one. Increasing values indicate a more complex shape, and more contact between the patch and its surroundings. The proximity index of forest patches relates to the amount of forest within a specified radius around a patch,

Table 1 Variables selected in the hazard or exposure model

\begin{tabular}{|c|c|c|c|c|c|}
\hline & Hazard & Exposure & Resolution & Units & Sources \\
\hline Roe deer/reddeer/fallow deer/wild boar & $x$ & & $\begin{array}{l}\text { Low (interpolation based on } \\
\text { hunting districts centers) }\end{array}$ & $\begin{array}{l}\text { Number of animals } \\
\text { per hectar }\end{array}$ & Dr. Jonas Kindberg \\
\hline Proportion of forest in the buffer & $x$ & $x$ & $100 \mathrm{~m}$ & Percentage & CORINE \\
\hline Proportion of broad-leaved forest in the buffer & $x$ & $x$ & $100 \mathrm{~m}$ & Percentage & CORINE \\
\hline Proportion of coniferous forest in the buffer & $x$ & & $100 \mathrm{~m}$ & Percentage & CORINE \\
\hline Proportion of mixed forest in the buffer & $x$ & & $100 \mathrm{~m}$ & Percentage & CORINE \\
\hline Shape index of forest in the buffer & $x$ & & $100 \mathrm{~m}$ & None & CORINE \\
\hline $\begin{array}{l}\text { Mean proximity index for forest patches } \\
\text { in the buffer }\end{array}$ & $x$ & & $100 \mathrm{~m}$ & None & CORINE \\
\hline $\begin{array}{l}\text { Mean volume of spruce/pine/birch/oak } \\
\text { in the buffer }\end{array}$ & $x$ & & $30 \mathrm{~m}$ & $\mathrm{~m}^{3} / \mathrm{ha}$ & SLU skogskarta \\
\hline $\begin{array}{l}\text { Proportion of clear-cuts (tree height }<50 \mathrm{~cm} \text { ) } \\
\text { in the forest in the buffer }\end{array}$ & $x$ & $x$ & $30 \mathrm{~m}$ & Percentage & SLU skogskarta \\
\hline Proportion of waterbodies in the buffer & $x$ & $x$ & $100 \mathrm{~m}$ & Percentage & CORINE \\
\hline Distance to the nearest water course & $x$ & $x$ & High (shapefile) & $\mathrm{m}$ & Lantmäteriet \\
\hline $\begin{array}{l}\text { Proportion of open areas in ecotone of } 150 \mathrm{~m} \\
\text { around forest in the buffer }\end{array}$ & $x$ & & $100 \mathrm{~m}$ & Percentage & CORINE \\
\hline Shannon diversity index in the buffer & $x$ & & $100 \mathrm{~m}$ & None & CORINE \\
\hline Length of roads in the buffer & & $x$ & High (shapefile) & $\mathrm{m}$ & Lantmäteriet \\
\hline Length of roads in forest in the buffer & & $x$ & High (shapefile) & $\mathrm{m}$ & Lantmäteriet \\
\hline Distance to Stockholm & & $x$ & High (shapefile) & $\mathrm{m}$ & Lantmäteriet \\
\hline $\begin{array}{l}\text { Proportion of area occupied by holiday } \\
\text { houses in the buffer }\end{array}$ & & $x$ & High (shapefile) & Percentage & Statistiska Centralbyrån \\
\hline Mean population density & & $x$ & 2.5 arc-minutes & Person $/ \mathrm{km}^{2}$ & $\begin{array}{l}\text { Gridded Population } \\
\text { of the World }\end{array}$ \\
\hline Distance to the sea & & $x$ & High (shapefile) & $\mathrm{m}$ & Lantmäteriet \\
\hline Standard deviation of tree height in the buffer & & $x$ & $30 \mathrm{~m}$ & $\mathrm{~m}$ & SLU skogskarta \\
\hline Mean height tree & & $x$ & $30 \mathrm{~m}$ & $\mathrm{~m}$ & SLU skogskarta \\
\hline
\end{tabular}


and indicates whether a patch is isolated or fragmented.

Also, as various tree species may impact tick habitat suitability differently, the mean volume of spruce, oak, birch and pine per hectare in the buffer were added (30 meter resolution, SLU Skogskarta, Swedish University of Agricultural Sciences). Forest areas where tree height was lower than 50 centimeters were used as a proxy to represent clearcuts (SLU Skogskarta). Intensive clear-cutting is non-valuable for wildlife but, in the study area, clear-cuts were small (mean area of $1682.68 \mathrm{~m}^{2}$ and mean cross-section of $146.54 \mathrm{~m}$ ). The area of clearcuts was divided by the area of forest in the buffer. While clear-cuts may provide food for various host species, it does not provide as good shelter as forests.

iii. Land cover

Forest ecotones, particularly where they connect to open areas, can be very suitable for ticks and hosts as these habitats offer a high diversity of resources [16]. The main roe deer habitat is also deciduous or mixed forest with open areas $[13,15]$. The proportion of open areas (agricultural and transitional area from CORINE) in ecotones of 150 meters around forests in the buffer was added to the hazard model.

The Shannon diversity index, representing the richness of the landscape in the buffer, was included [17].

Since high humidity favours tick questing, moist areas are more suitable for ticks [16]. The proportion of waterbodies in the buffer (CORINE) and the distance to the nearest water course (Lantmäteriet) were included as proxies for moister areas.

\section{Variables describing exposure}

Variables describing the exposure, the degree to which people enter infected landscape, were identified through the scientific literature studying landscape attractiveness for touristic activities (Table 1).

\section{i. Accessibility}

A study of tourist preferences indicated that accessibility to forest increases the touristic value of forest [18]. Indeed, in Sweden, there is a traditional right of public access to private land, e.g. to enter forests and to harvest resources such as mushrooms and berries $[18,19]$. Assuming that roads increase access, and that forests with roads are more likely to be entered by visitors, we included the length of roads in the buffer (Lantmäteriet) and the length of roads in forests in the buffer to describe accessibility.
Assuming that places with holiday cabins would relate to outdoor activities, the area occupied by holiday houses in the buffer was included (Statistics Sweden (Statistiska Centralbyrån)). In Sweden, 50 of holiday houses are within a radius of 32 kilometres from permanent homes [20]. The distance to Stockholm, from which many holiday cabin users originate, was included in the model (Lantmäteriet database), assuming that areas closer to Stockholm would be more frequently used for outdoor recreation. Population density (2.5 arc-minutes resolution, Gridded Population of the World from Center for International Earth Science Information Network (CIESIN)) was also included.

ii. Scenic beauty Landscape features documented to increase the perceived scenic beauty include water features [21] and broad-leaved forests [18]. The distance to the nearest water course (Lantmäteriet database), proportion of waterbodies in the buffer (CORINE), and the proportion of forest and of broad-leaved forest (CORINE) were used. In Finland, a preference for forest stands with a higher mean tree height and a skewed distribution of height has been highlighted [22]. The mean tree height in the buffer was calculated and standard deviation of tree height was used as a proxy for the skewness (SLU Skogskarta). In Sweden, the touristic value of a forest increases with the number of clear-cuts and decreases with the size of the clear-cuts within a given area [18]. The proportion of clear-cuts in the forest (SLU Skogskarta) in the buffer was thus added to the exposure dataset.

\section{Methods}

\section{Principal component analyses}

The potential explanatory variables outlined above are numerous, mostly proxies, and sometimes redundant. Therefore, principal component analyses (PCA) were used to identify sub-groups of similar variables ("Rcmdr" package and plugin "FactoMineR" in R 2.12.0). The factorial coordinates were used as new variables. Two variables were selected: one summarizing the variables on wildlife species (wild boar, red deer, fallow deer and roe deer) and another, accessibility variables (population density, length of roads, distance to Stockholm and length of roads in forest).

\section{Boosted regression trees}

The multivariate models were built using boosted regression trees (BRT) ("gbm" package in R) [23]. BRT have been identified as an efficient method for investigating variables explaining the spatial distribution of zoonotic diseases [24]. A major advantage compared to regression is that $\mathrm{BRT}$ allows the modelling non-linear 
responses. BRT results comprises of two essential elements: relative importance and response curve. The relative importance of each variable represents the number of times a variable was used in successive trees, weighted by the mean of the squared improvement provided by this variable to each tree [23]. Response curves are graphs representing the evolution of the fitted probability function according to the variation of the variable. They were interpreted here as a relative probability of being in the presence of the disease at various levels of the predictor variable.

In a BRT, an internal node represents a variable that is cutting the data into several branches that lead to other nodes [23]. The decision of presence or absence is made at terminal nodes. The new trees are fitted on the residuals of the previous trees and the new model contains both previous and new trees. At each step, 50 of the data are randomly selected to enlarge the previous trees. The learning rate (contribution of each tree to the final model), the tree complexity (number of nodes in a tree) and the number of trees are chosen in order to optimize the predictive power.

Three models were built containing respectively the hazard variables, the exposure variables, and both hazard and exposure in a global model. Some variables were included in both hazard and exposure models as they may relate to either aspect (Table 1). To account for potential spatial structure in the distribution of TBE cases, the proportion of infected places within a radius of $20 \mathrm{~km}$ was added to each model.

\section{Measures of the predictive power}

\section{Internal validation of the predictive power}

As BRT builds the trees on random subsamples, each model (hazard, exposure and global) was run 25 times. The mean areas under the curve (AUC) ("PresenceAbsence" package in R) were compared using a Student ttest. An AUC of 0.5 indicates a random distribution of predictions, and of 1 a perfect prediction [25,26]. False presences and absences, using the sensitivity equals the specificity as probability threshold, were mapped.

\section{External validation of the predictive power}

External AUC for each model were calculated from cross-validation on 10 subsets. Models were run on nine subsamples and AUC was calculated on a tenth subsample. This step is repeated 10 times, using a different validation sub-sample each time. The final AUC is the mean of the AUC calculated on the 10 evaluation subsamples. This was run 25 times and compared with a Student t-test.

Moreover, TBE records from 2011 were used to assess the predictive power of our models. Continuous predicted probability maps were created for each model by kriging, on which TBE presence records in 2011 were overlaid.

The presence in 2011 were completed with absences (settlements with no presence records between 19982007 and in 2011). Then, predicted probabilities were calculated for this new dataset. The means of predicted probabilities located at presence points were compared to absences points by a Welch test.

\section{Results}

\section{Principal component analyses}

Two PCAs were computed. The two first components of the PCA on the data on wildlife species explained 71.76 of the variance. The first component (variable PC1: Wildlife) was positively correlated with wild boars (correlation of 0.85), red deer (0.84) and fallow deer (0.64). The second component was only positively correlated with roe deer (0.99), which was subsequently kept as an individual variable. The first two components of the PCA on accessibility variables explained 66.36 of the variance. The first component (variable PC1: Accessibility) was positively correlated with the human population density (correlation of 0.76), the length of roads (0.65) and negatively correlated with the distance to Stockholm $(-0.75)$. The second dimension was positively correlated with the length of roads in the forest $(0.92)$ and was also kept as an individual variable.

\section{Boosted regression trees}

The variables with the highest relative importance (variables forming the first 50 of summed relative importance) in the hazard model were the number of TBE cases within $20 \mathrm{~km}$ (relative importance of 23.23), volume of spruce (10.71), distance to a water course (7.30), total proportion of forest (5.40) and proportion of coniferous forest (5.33) (Table 2).

In the exposure model, the variables with the highest relative importance were the number of TBE cases within $20 \mathrm{~km}$ (relative importance of 25.42), length of roads in forest (17.67) and distance to a water course (7.79) (Table 2).

The trends of the variable response curve according to the probability of finding TBEV were similar in the tree models. Response curve graphs of the global model are represented in Figure 2. Variables which showed a global positive trend are: infections in $20 \mathrm{~km}$ (relative importance of 17.94); roads in forest (11.56), holiday houses (4.94), PC1: Accessibility (4.16), oak (3.76), birch (3.50), Shannon index (3.19), forest shape index (3.17), mixed forest (3.00), clear-cuts (2.88), standard deviation of tree height (2.71), forest proximity index (2.07), broad-leaved forest (1.52) and, waterbodies (0.97). Variables that showed a global negative trend are: spruce (8.10), coniferous (3.84), mean trees height (2.78) and roe deer 
Table 2 Relative importance of variables introduced in the hazard and in the exposure boosted regression trees

\begin{tabular}{|c|c|c|c|}
\hline \multicolumn{2}{|l|}{ Hazard model } & \multicolumn{2}{|l|}{ Exposure model } \\
\hline Variable & Relative importance (\%) & Variable & Relative importance (\%) \\
\hline Infections in $20 \mathrm{~km}$ & 23.23 & Infection in $20 \mathrm{~km}$ & 25.42 \\
\hline Volume of spruce & 10.71 & Length of roads in forest & 17.67 \\
\hline Distance to water course & 7.30 & Distance to water course & 7.79 \\
\hline Volume of oak & 6.33 & Proportion of forest & 7.75 \\
\hline Proportion of forest & 5.40 & Mean height of trees & 7.09 \\
\hline Proportion of coniferous & 5.33 & PC1: Accessibility & 6.78 \\
\hline Proportion of clear-cuts & 5.28 & Proportion of holiday houses & 6.18 \\
\hline Volume of birch & 5.24 & Distance to the sea & 5.49 \\
\hline Forest shape index & 5.04 & Standard deviation of height of trees & 5.19 \\
\hline Shannon diversity index & 4.83 & Proportion of clear-cuts & 5.19 \\
\hline Volume of pine & 4.47 & Proportion of broad-leaved forest & 3.74 \\
\hline Proportion of mixed forest & 4.26 & Proportion of waterbodies & 1.72 \\
\hline Forest proximity index & 3.60 & & \\
\hline Open areas in ecotones & 3.05 & & \\
\hline Proportion of broad-leaved forest & 2.20 & & \\
\hline PC1: Wildlife & 1.31 & & \\
\hline Proportion of waterbodies & 1.31 & & \\
\hline Roe deer & 1.05 & & \\
\hline
\end{tabular}

(1.35). Variables which showed an important decrease followed by an increase are: distance to water course (4.55), distance to the sea (4.15), forest (3.70), open area in ecotone (2.31) and, PC1: Wildlife (0.74). Pine (3.12) showed a first peak around $15 \mathrm{~m}^{3} /$ ha followed by an increase around $70 \mathrm{~m}^{3} / \mathrm{ha}$.

\section{Measures of the predictive power}

The mean AUC were 0.92 for the hazard and exposure models and 0.93 for the global model. AUC were lower for the cross-validated data with 0.74 for the hazard and exposure models and 0.75 for the global model. The mean AUC of global models are significantly higher (pvalue $<0.001$ ) than the mean AUC of hazard models and exposure models.

There were few false absences: 18 for the hazard model, 30 for the exposure model and 18 for the global model (Figure 3). There were more false presences: 681 for the hazard model, 482 for the exposure model and 593 for the global model (Figure 3).

The false presence of the hazard models were in areas within the TBEV focus while false presences of exposure model were distributed in areas where disease cases have not yet been recorded (Figure 3). The global model had the best visual match between areas with high interpolated probabilities and high frequency of presence in 2011. The hazard and exposure models both appear to contribute to the distribution of high probability areas observed in the global model map (Figure 3).

The mean probability predicted by the global model on presence in $2011\left(44.05 * 10^{-3}\right)$ was significantly different ( $p$-value $<0.001$ ) from the mean probability of absence $\left(27.51 * 10^{-3}\right)$. Similar results were observed for probabilities extracted from the hazard and exposure models.

\section{Discussion}

\section{Comparison of hazard and exposure}

All three models, focusing on hazard, exposure and all factors, respectively, reached a good fit and a reasonable ability to predict hot spots for an independent year, i.e. 2011. However, the global model was clearly the most exhaustive in indicating areas of higher probabilities. Both the aspects of hazard and exposure therefore deserve consideration when examining risk and its spatial distribution. The two variables with the highest relative importance in the global model, other than the variable describing spatial structure, were the roads in forest positively related to the exposure, and the volume of spruce negatively related to the hazard, underlining the importance of accounting for both aspects of risk. This makes perfect sense when approaching the question of the spatial distribution of a zoonotic disease using human case records: human land use is spatially heterogeneous. Beyond the need to account for all factors 


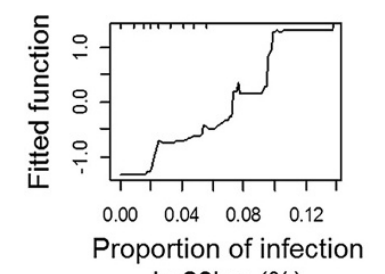

in $20 \mathrm{~km}(\%)$
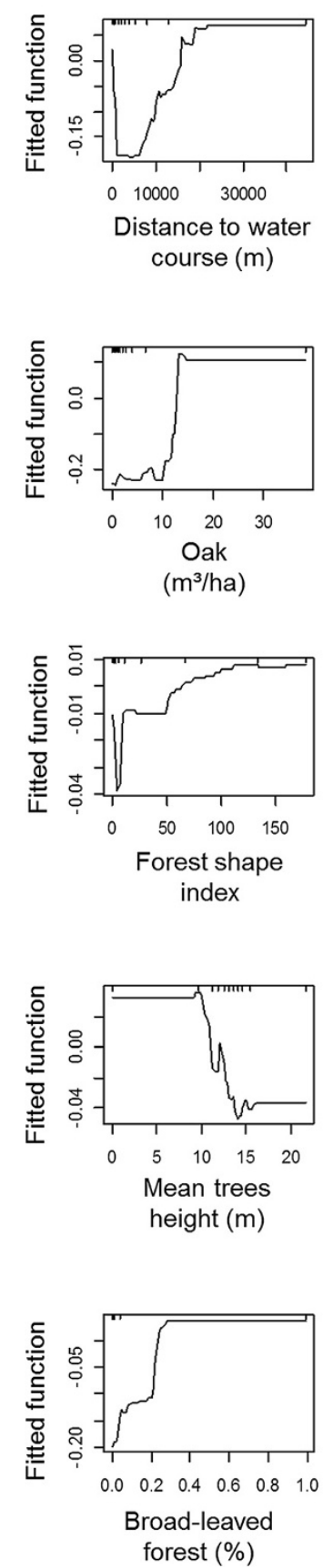

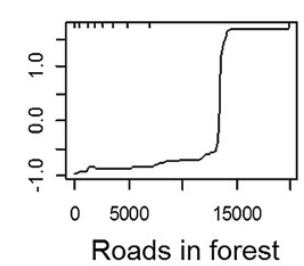

(m)
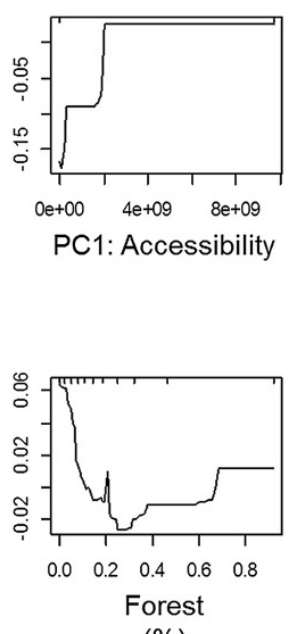

(\%)
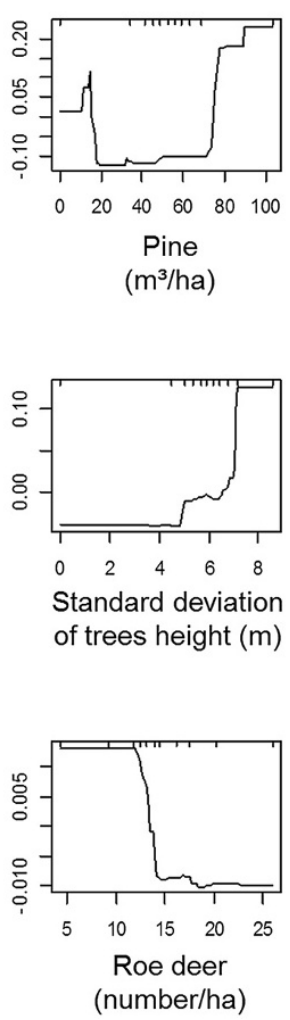
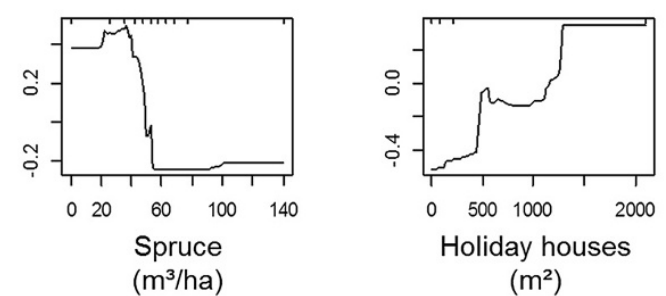

$\left(\mathrm{m}^{2}\right)$

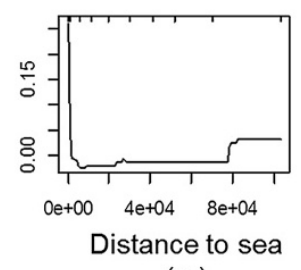

(m)
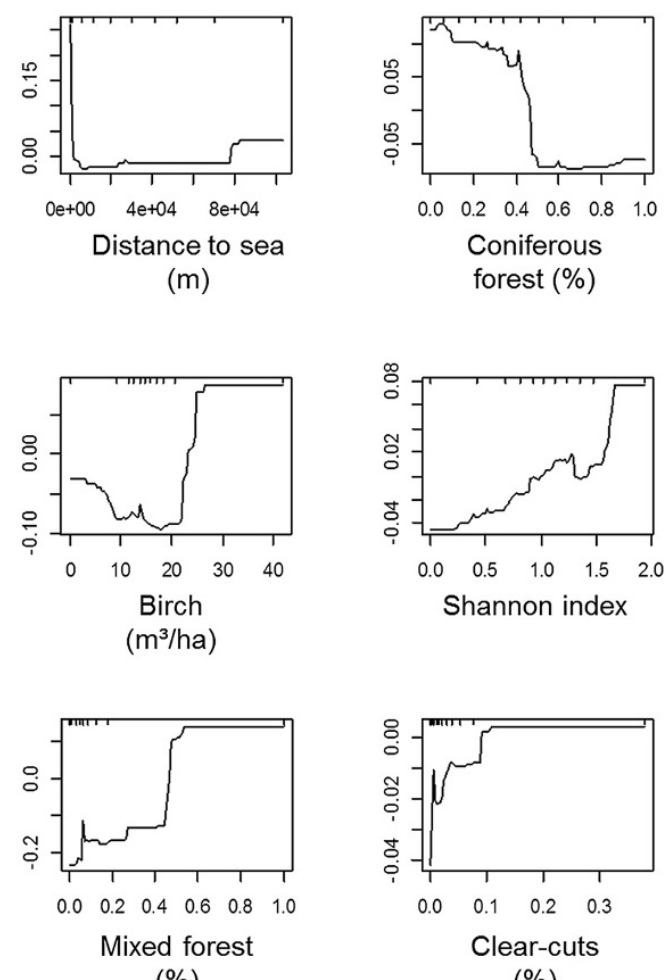

(\%)

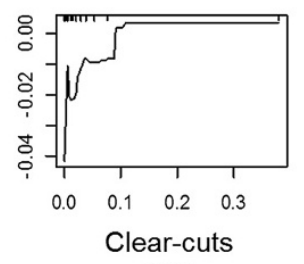

(\%)
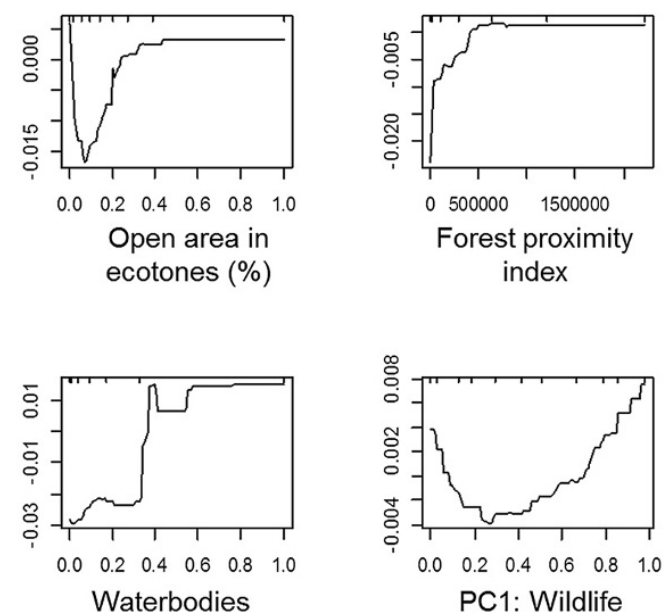

(\%)

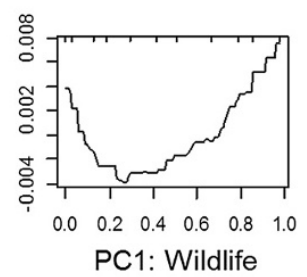

Figure 2 Graphs of each variable according to the fitted function of the global model (percentage represents the relative importance of the variable). 


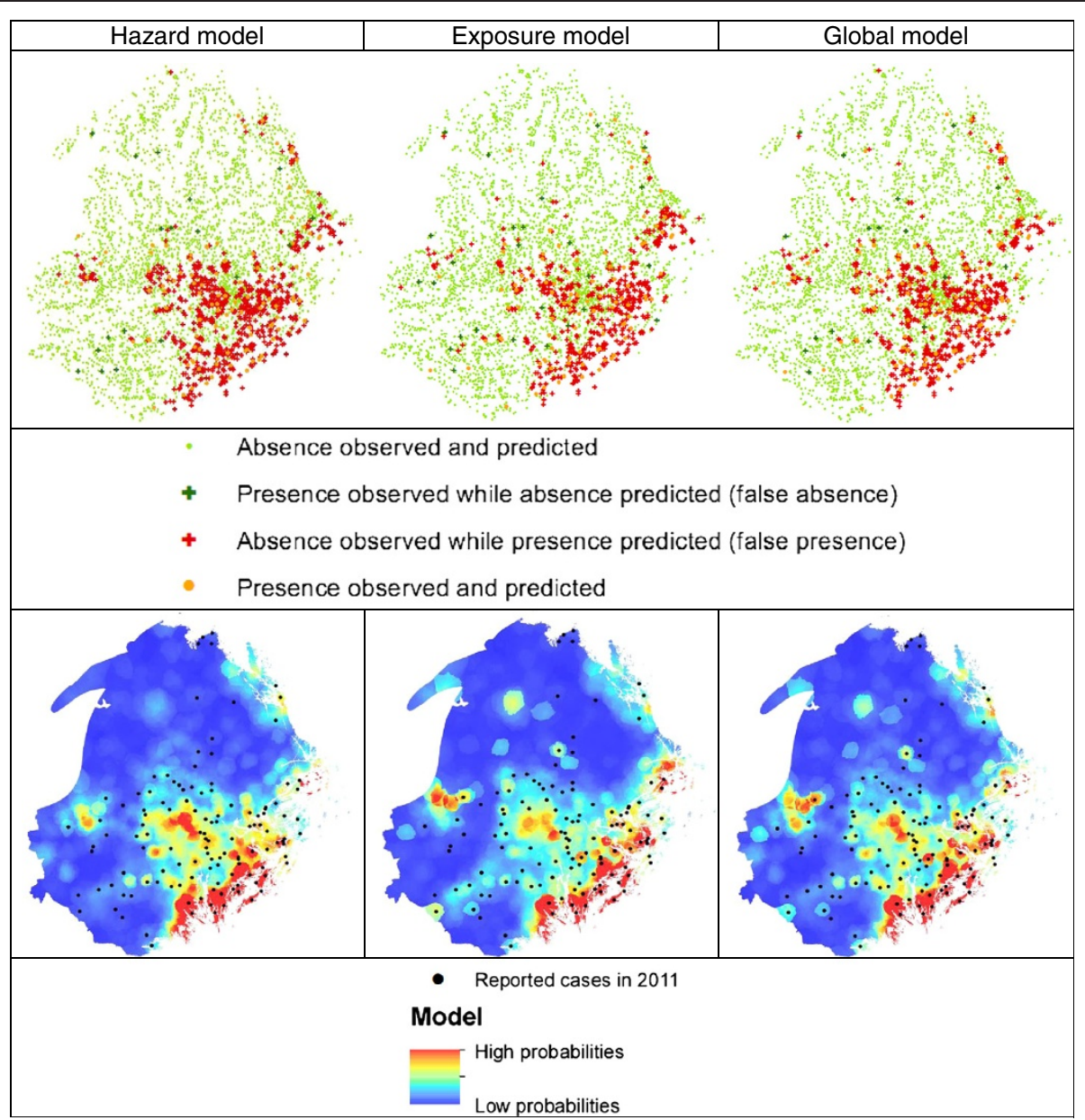

Figure 3 Resulting maps of the hazard, exposure and global models (based on 1998-2007 TBE records) and TBE records in 2011.

explaining the spatial distribution, factors related to exposure may offer important keys for the understanding of zoonotic disease emergence and human risk. In this study, the spatial distribution of TBE cases in Sweden looked beyond hazard-related factors and classic epidemiologic factors such as occupation to include variables depicting specifically where people at risk are most likely to enter infested areas.

False presences identified for the various models did not follow the same spatial pattern (Figure 3). These false presences could be: models errors; locations suitable for transmission but where the pathogen or susceptible humans are absent; areas where the pathogen is circulating but not transmitted to humans and; areas where the disease is found but not recorded (non-identified, nonreported or asymptomatic cases). Considering false presence as points were TBEV may appear in the near future, the exposure model seems suitable for predicting the disease in new areas. Inversely, looking at the probability maps, the hazard model seems to show a better prediction in areas of spatially concentrated TBE infectious areas and so a better prediction of intensification of the disease. There are still a few points outside the high probability areas, indicating that some variables may be missing in the models.

Challenges related to this hazard/exposure approach relate essentially to the interpretation of variables as influencing hazard or exposure. Some variables may be related to hazard or exposure in an unambiguous way, but several may be proxy for both vector (or host) habitat and landscape attractiveness for human. For example, while tree species would presumably relate to hazard, a study conducted in Finland indicates that touristic preferences increase with the volume of pines and birches [22]. Scale may influence the interpretation of variables as related to hazard or exposure. For example, the distance to the sea, here used in the exposure model, may also influence, at broader scale, the length of the 
vegetation season and the suitability for vectors and hosts through its buffering effect on temperature [14]. Careful consideration of the interpretation and scale of variables included in risk, hazard or exposure models is therefore necessary.

Both hazard and exposure variables are needed for a better understanding of the spatial distribution of vector-borne diseases, but exposure variables may be specific to regions, just as some epidemiological risk factors can be culturally driven. Sweden, for instance has a particular public right of access to land ("Allemansrätten"). Still, accessibility is not evenly developed everywhere, and other factors may influence accessibility or attractiveness, such as land ownership [27].

\section{Variables influencing the distribution of TBE in Sweden}

This study highlights the main spatial variables influencing the distribution of TBE in a highly endemic region of Sweden. Forests with the highest probabilities of presence were well-connected oak, birch and pine forests, with a complex shape, numerous clear-cuts and a tree height variation of at least five meters. Landscapes with the highest probabilities were easy to reach, with high landscape diversity (Shannon index), holiday houses, waterbodies and broad-leaf or mixed forest with open area in their ecotones. Fitted function curves mostly follow our preliminary assumptions based on the literature or field experience. Therefore, spruce forests are less favourable than pine forests, probably because they have less undergrowth. Also, the high probability in very close proximity to the sea is related to the presence of houses near the shore. The results for the distance to water course raise new questions as it is a less prominent feature of the landscape than waterbodies.

Few interactions were identified. The most important interaction was between areas of holiday houses, a proxy for attractiveness, and places where there were more than $10 \mathrm{~km}$ of roads in forest, a proxy for accessibility. Furthermore, correlation between variables makes some probability distributions difficult to interpret. For instance, tree height, which is positively correlated to the proportion of coniferous trees, is negatively correlated to broad-leaved-forest. Decreasing probabilities with the tree height may thus relate to the decreasing probabilities with conifers. Also, low pine volume may imply the presence of larger volumes of deciduous trees and explain the peak around $17 \mathrm{~m}^{3} / \mathrm{ha}$.

Probabilities of TBE cases increased with the first PCA component of wildlife (positively correlated with wild boars, red deer and fallow deer) and decreased with the abundance of roe deer. Deer and wild boars (and, maybe, more specifically, young wild boars) most likely constitute important blood meal sources for adult female ticks before egg laying. A negative response of TBE with roe deer was previously highlighted in Sweden [13] and, at a local scale, in Italy and Slovakia, with the increase of cofeeding ticks on rodent when deer density is decreasing [28]. Both studies hypothesized a dilution effect due to a high density of deer (incompetent hosts) diverting the questing ticks from rodents (competent hosts). However, a mathematical model estimating the threshold for tickborne disease persistence reveals that, in the case of non-viraemic transmission, the dilution effect is less relevant [29]. Here, the PCA reflects that roe deer are not found in the same places as the three other species. The decreasing probability may therefore result not from roe deer specifically, but from unsuitability for any aspect of the transmission cycle. Further investigation on the role of wildlife in feeding ticks and hosting the TBE virus would be useful. These results highlight the need for a better understanding of the TBEV transmission system and the mechanisms underlying statistical relationship. Only in this way could such results be meaningful for risk prediction and public health.

\section{Conclusions}

Our study of the distribution of human cases of TBE in Sweden indicates that separating and accounting specifically for hazard and exposure in distribution models holds great potential for the understanding and the mapping of zoonotic disease spatial pattern and emergence. Exposure variables were extracted from standard GIS data bases following a similar strategy as is classically done for studies focusing on the hazard.

TBE is emerging in different places in Europe and understanding this pattern is essential to help public health. Randolph compares human cases to "the tip of the iceberg" that emerges from the undetected enzootic cycles below the surface [3]. As ecological processes driving the distribution of TBEV are not yet completely described, it is of great value to be able to track the sources of human TBE back to infection sites, trying to unravel the role of local wildlife on the persistence and circulation of TBEV. Accounting for exposure may also contribute to this by allowing more specific interpretation of any variable in the model.

In conclusion, linking ecology and public health is highly recommended. While the conditions for access and use of hazardous areas highlighted in this study may be specific to Scandinavia, this unified method offers promising perspectives to further understand the distribution of various zoonotic and vector-borne diseases in diverse contexts by the explicit inclusion of exposurerelated variables.

\section{Abbreviations}

TBE: Tick-borne encephalitis; TBEV: Tick-borne encephalitis virus; PCA: Principal component analysis; BRT: Boosted regression trees; AUC: Area under the curve. 


\section{Competing interests}

The authors declare that they have no competing interests.

\section{Authors' contributions}

CZ and SV designed the study, carried out the spatial and statistical analysis, interpreted the results and drafted the manuscript. GO helped in the selection of variables, interpreted the results and critically revised the manuscript. $\mathrm{MH}$ collected the data, interpreted the results and critically revised the manuscript. All authors read and approved the final manuscript.

\section{Acknowledgments}

This study was funded by EU grants FP7-261504 EDENext and is catalogued by the EDENext Steering Committee as EDENext180 (http://www.edenext.eu). The contents of this publication are the sole responsibility of the authors and don't necessarily reflect the views of the European Commission. The authors thank Jonas Kindberg for the data on bag statistics and for sharing his knowledge on the ecology of the study area.

\section{Author details}

${ }^{1}$ Earth and Life Institute, Georges Lemaitre Centre for Earth and Climate Research (TECLIM), Université Catholique de Louvain (UCL), GEOG, Place Louis Pasteur 3 bte L4.03.07, 1348 Louvain-la-Neuve, Belgium. ²Department of Wildlife, Fish, and Environmental Studies, Swedish University of Agricultural Sciences (SLU), Umea, Sweden. ${ }^{3}$ Swedish Institute for Communicable Disease Control (SMI), Stockholm, Sweden.

Received: 14 April 2014 Accepted: 31 July 2014

Published: 15 August 2014

\section{References}

1. Jones KE, Patel NG, Levy MA, Storeygard A, Balk D, Gittleman JL, Daszak P: Global trends in emerging infectious diseases. Nature 2008, 451:990-993.

2. FAO: Principles and guidelines for the conduct of microbiological risk assessment. Codex Alimentarius: Food and hygiene basic texts. Rome: Food and Agriculture Organization of the United Nations; 1999:53-62.

3. Randolph SE: Tick-borne encephalitis virus, ticks and humans: Short-term and long-term dynamics. Curr Opin Infect Dis 2008, 21:462-467.

4. Stefanoff P, Rosinska M, Samuels S, White DJ, Morse DL, Randolph SE: A National Case-control Study Identifies Human Socio-Economic Status and Activities as Risk Factors for Tick-Borne Encephalitis in Poland. PLoS One 2012, 7:e45511.

5. Dumpis U, Crook D, Oksi J: Tick-borne encephalitis. Clin Infect Dis 1999, 28:882-890.

6. Parola P, Raoult D: Ticks and tickborne bacterial diseases in humans: An emerging infectious threat. Clin Infect Dis 2001, 32:897-928.

7. Randolph SE: Transmission of tick-borne pathogens between co-feeding ticks: Milan Labuda's enduring paradigm. Ticks Tick Borne Dis 2011, 2:179-182.

8. Randolph SE, Gern L, Nuttall PA: Co-feeding ticks: Epidemiological significance for tick-borne pathogen transmission. Parasitol Today 1996, 12:472-479.

9. Randolph SE, Miklisová D, Lysy J, Rogers DJ, Labuda M: Incidence from coincidence: Patterns of tick infestations on rodents facilitate transmission of tick-borne encephalitis virus. Parasitology 1999, 118:177-186.

10. Lundkvist A, Wallensten A, Vene S, Hjertqvist M: Tick-borne encephalitis increasing in Sweden, 2011. Euro Surveill 2011, 16:39.

11. Jaenson TGT, Jaenson DGE, Eisen L, Petersson E, Lindgren E: Changes in the geographical distribution and abundance of the tick Ixodes ricinus during the past 30 years in Sweden. Parasit Vectors 2012, 5:8.

12. Medlock JM, Hansford KM, Bormane A, Derdakova M, Estrada-Peña A, George JC, Golovljova I, Jaenson TGT, Jensen JK, Jensen PM, Kazimirova M, Oteo JA, Papa A, Pfister K, Plantard O, Randolph SE, Rizzoli A, Santos-Silva MM, Sprong H, Vial L, Hendrickx H, Zeller H, Van Bortel W: Driving forces for changes in geographical distribution of Ixodes ricinus ticks in Europe. Parasit Vectors 2013, 6:1.

13. Jaenson TGT, Hjertqvist M, Bergström T, Lundkvist $\AA$ : Why is tick-borne encephalitis increasing? A review of the key factors causing the increasing incidence of human TBE in Sweden. Parasit Vectors 2012, 5:184.

14. Jaenson TGT, Eisen L, Comstedt P, Mejlon HA, Lindgren E, Bergström S, Olsen B: Risk indicators for the tick Ixodes ricinus and Borrelia burgdorferi sensu lato in Sweden. Med Vet Entomol 2009, 23:226-237.
15. Tack W, Madder M, Baeten L, De Frenne P, Verheyen K: The abundance of Ixodes ricinus ticks depends on tree species composition and shrub cover. Parasitology 2012, 139:1273-1281.

16. Lindström A, Jaenson TGT: Distribution of the common tick, Ixodes ricinus (Acari: Ixodidae), in different vegetation types in southern Sweden. J Med Entomol 2003, 40:375-378.

17. Shannon C, Weaver W: The mathematical theory of communication. Urbana: University of Illinois Press; 1949.

18. Bostedt G, Mattsson L: The value of forests for tourism in Sweden. Ann Tour Res 1995, 22:671-680.

19. Lindhjem $\mathrm{H}: 20$ years of stated preference valuation of non-timber benefits from Fennoscandian forests: A meta-analysis. J For Econ 2007, 12:251-277.

20. Müller DK: The attractiveness of second home areas in Sweden: A quantitative analysis. Curr Issues Tourism 2006, 9:335-350.

21. Nielsen AB, Heyman E, Richnau G: Liked, disliked and unseen forest attributes: Relation to modes of viewing and cognitive constructs. J Environ Manage 2012, 113:456-466.

22. Silvennoinen $\mathrm{H}$, Alho J, Kolehmainen O, Pukkala T: Prediction models of landscape preferences at the forest stand level. Landsc Urban Plan 2001, $56: 11-20$.

23. Elith J, Leathwick J, Hastie T: A working guide to boosted regression trees. J Anim Ecol 2008, 77:802-813.

24. Zeimes CB, Olsson GE, Ahlm C, Vanwambeke SO: Modelling zoonotic diseases in humans: comparison of methods for hantavirus in Sweden. Int J Health Geogr 2012, 11:39.

25. Pearce J, Ferrier S: Evaluating the predictive performance of habitat models developed using logistic regression. Ecol Model 2000, 133:225-245.

26. Fawcett T: An introduction to ROC analysis. Pattern Recognit Lett 2006, 27:861-874.

27. Vanwambeke SO, Šumilo D, Bormane A, Lambin EF, Randolph SE: Landscape predictors of tick-borne encephalitis in Latvia: Land cover, land use, and land ownership. Vector-Borne Zoonot Dis 2010, 10:497-506.

28. Cagnacci F, Bolzoni L, Rosà R, Carpi G, Hauffe HC, Valent M, Tagliapietra V, Kazimirova M, Koci J, Stanko M, Lukan M, Henttonen H, Rizzoli A: Effects of deer density on tick infestation of rodents and the hazard of tick-borne encephalitis. I: Empirical assessment. Int J Parasitol 2012, 42:365-372.

29. Rosà R, Pugliese A, Norman R, Hudson PJ: Thresholds for disease persistence in models for tick-borne infections including non-viraemic transmission, extended feeding and tick aggregation. J Theor Biol 2003, 224:359-376.

doi:10.1186/1756-3305-7-370

Cite this article as: Zeimes et al: Shaping zoonosis risk: landscape ecology vs. landscape attractiveness for people, the case of tick-borne encephalitis in Sweden. Parasites \& Vectors 2014 7:370.

\section{Submit your next manuscript to BioMed Central and take full advantage of:}

- Convenient online submission

- Thorough peer review

- No space constraints or color figure charges

- Immediate publication on acceptance

- Inclusion in PubMed, CAS, Scopus and Google Scholar

- Research which is freely available for redistribution

Submit your manuscript at www.biomedcentral.com/submit
C Biomed Central 